\title{
A Dynamic Programming Algorithm for Energy-aware Routing of Delivery Drones
}

\author{
Yusuke Funabashi $^{1}$ Atsuya Shibata ${ }^{1}$ Shunsuke Negoro $^{1}$ Ittetsu Taniguch $^{2, \text { a) }}$ \\ Hiroyuki TOMIYAMA ${ }^{1, b)}$ \\ Received: November 29, 2019, Revised: February 14, 2020, \\ Accepted: April 23, 2020
}

\begin{abstract}
Energy consumption is one of the most critical concerns in delivery services using drones. This paper studies a routing problem for energy minimization of delivery drones. This paper formally defines Energy Minimizing Vehicle Routing Problem (EMVRP) and proposes a dynamic programming algorithm to efficiently solve the problem. Experiments show the effectiveness of the proposed algorithm in terms of both quality of results and algorithm runtime.
\end{abstract}

Keywords: EMVRP, dynamic programming, drone delivery

\section{Introduction}

Drones are promising vehicles for home delivery services since they are not affected by traffic jams. This paper addresses a kind of vehicle routing problem (VRP) for delivery drones. Given a set of items to deliver, the problem asks an optimal route which starts from a depot, delivers all of the items to customers, and comes back to the depot. VRP is similar to the traditional traveling salesman problem (TSP) which asks the shortest route in distance. On the other hand, the problem addressed in this paper asks the route with the minimum energy consumption, which is called an energy minimizing vehicle routing problem (EMVRP) [1], [2], [3], [4], since battery lifetime is one of the most critical concerns in delivery drones.

Let us consider an example shown in Fig. 1. The node labeled " 0 " denotes a depot, and the other three nodes denote customers (i.e., shipping destinations). The numbers in the boxes represent the weight of the items to deliver, and the numbers on the edges represent the distance between the two places. The optimal route for TSP is shown in Fig. 1 (a). The total distance of the route is $1,964(=362+468+665+469)$. However, this route may not be optimal for EMVRP. The energy consumption of drones depends not only on the flight distance but also on the total weight of loaded items. The heavier the load is, the higher the energy consumption is. This implies that heavy items should be delivered as early as possible. The optimal route for EMVRP is shown in Fig. 1 (b) ${ }^{* 1}$. The total distance of the EMVRP route is $2,015(=283+468+795+469)$, which is longer than the TSPoptimal route. Thus, the shortest route is not always same as the

Graduate School of Science and Engineering, Ritsumeikan University, Kusatsu, Shiga 525-8577, Japan

2 Graduate School of Information Science and Technology, Osaka University, Suita, Osaka 565-0871, Japan

a) i-tanigu@ist.osaka-u.ac.jp

b) ht@fc.ritsumei.ac.jp

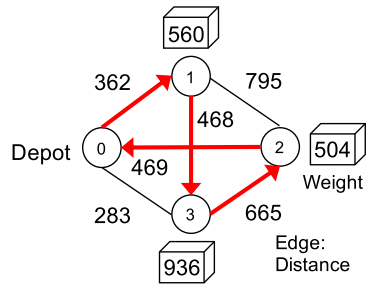

(a) TSP

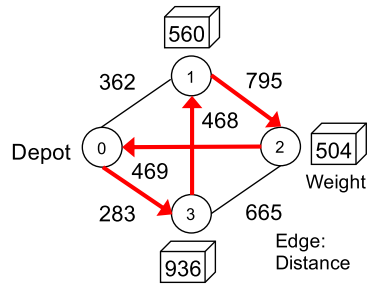

(b) EMVRP
Fig. 1 Optimal routes.

minimum-energy route.

This paper proposes a dynamic programming algorithm for EMVRP. The proposed algorithm efficiently finds exactly optimal routes in terms of energy consumption.

\section{The Routing Problem for Delivery Drones}

We are given $N$ items to deliver. Without loss of generality, no two items are to be delivered to the same customer. The items or customers are numbered from 1 to $N$. The customer where item $i$ $(1 \leq i \leq N)$ is to be delivered is called customer $i$. The depot is numbered 0 as shown in Fig. 1.

This paper assumes that the items are delivered in a single trip by a drone. All of the items are uploaded onto the drone at the depot, and the drone starts a trip. If the total weight of the items exceeds the capacity of the drone, the items need to be partitioned into groups before routing and delivery, and how to partition the items is out of the scope of this paper.

Let $w(i)$ denote the weight of item $i$ and $d(i 1, i 2)$ denote the distance between customers $i 1$ and $i 2$. Also, let $x(j)$ denote the $j$-th visited customer, which is the decision variable of the routing problem. Since a route starts and ends at the depot, we define:

$$
x(0)=x(N+1)=0
$$

\footnotetext{
*1 Actually, the optimal EMVRP route depends on the drone.
} 
Also, all of the customers are visited once, which is formally defined as follows:

$$
\begin{aligned}
& 1 \leq x(j) \leq N \quad(1 \leq j \leq N) \\
& x\left(j_{1}\right) \neq x\left(j_{2}\right) \quad\left(1 \leq j_{1}, j_{2} \leq N, j_{1} \neq j_{2}\right)
\end{aligned}
$$

Let $e(w, d)$ denote the energy consumption of the delivery drone, which is a function of payload weight $w$ and flight distance $d$. Function $e(w, d)$ depends on the drone, and is assumed to be given. For example, in Ref. [1], $e(w, d)$ is defined as

$$
e(w, d) \propto d \times\left(w+W_{\text {drone }}\right)
$$

where $W_{\text {drone }}$ is the weight of the drone itself. It should be noted that this work is not restricted to Formula (4).

Let $W(j)$ denote the total payload when the drone leaves $j$-th visited customer. When the drone starts a trip, all of the items are loaded. Therefore, the following formula holds.

$$
W(0)=\sum_{i=1}^{N} w(i)
$$

When the drone makes the $j$-th stop $(1 \leq j \leq N)$ at customer $x(j)$, an item of weight $w(x(j))$ is unloaded. Therefore, the total payload when the drone leaves customer $x(j)$ is defined as:

$$
W(j)=W(j-1)-w(x(j))
$$

Then, the objective function of our EMVRP is defined as:

$$
\text { minimize } E=\sum_{j=0}^{N} e(W(j), d(x(j), x(j+1)))
$$

The EMVRP addressed in this paper is formally defined as follows. Given $w, d$ and $e$, find $x$ which minimizes the objective function (7) while meeting the constraints (1)-(3), (5), (6) and (7).

\section{Dynamic Programming Algorithm}

This section outlines our dynamic programming (DP) algorithm for EMVRP*2. Let $\mathbb{S}$ denote a set of customers who are already visited, and let $i$ be the last-visited customer in $\mathbb{S}$. We call a pair $(\mathbb{S}, i)$ as a state. Obviously, the initial state is $(\phi, 0)$. Then, we define a problem asking the minimum energy consumption $E(\mathbb{S}, i)$ for delivery from the initial state to state $(\mathbb{S}, i)$. Now, we can derive a recurrence formula to calculate $E(\mathbb{S}, i)$ as follows.

$$
\begin{array}{r}
E(\mathbb{S}, i)=\min \left\{E\left(\mathbb{S} \backslash i, i^{\prime}\right)+e\left(W^{\prime}(\overline{\mathbb{S}})+w(i), d\left(i^{\prime}, i\right)\right)\right. \\
\left.\mid i^{\prime} \in \mathbb{S} \backslash i\right\}
\end{array}
$$

Recall that $i$ is the latest customer in $\mathbb{S}$. In the formula, $i^{\prime}$ denotes the second latest customer. $E\left(\mathbb{S} \backslash i, i^{\prime}\right)$ is the minimum energy consumption for flying from the depot to $i^{\prime}$, and $e\left(W^{\prime}(\overline{\mathbb{S}})+w(i), d\left(i^{\prime}, i\right)\right)$ is the energy consumption for flying from $i^{\prime}$ to $i$. $W^{\prime}(\overline{\mathbb{S}})$ denotes the total weight of items which are not yet delivered, which is formulated as:

$$
W^{\prime}(\overline{\mathbb{S}})=\sum_{k \notin \mathbb{S}} w(k)
$$

In Formula (8), it should be noted that, when departing from $i^{\prime}$,

\footnotetext{
*2 Due to the limited space, pseudo-code of the algorithm is not presented.
}

item $i$ is still loaded on the drone. Therefore, $w(i)$ is added to $W^{\prime}(\overline{\mathbb{S}})$. Also, it is obvious that the energy consumption at the initial state, i.e., before leaving the depot, is zero.

$$
E(\phi, 0)=0
$$

The original routing problem asks the minimum energy consumption when the drone departs from the depot, visits all of $N$ destinations, and comes back to the depot. Formally, the original problem asks:

$$
E(\{0,1,2, \ldots, N\}, 0)
$$

The problem in Expressoin (11) is recursively partitioned into sub-problems according to Formula (8), reaching Formula (10), and then, the optimal route with the minimum energy consumption is obtained.

\section{Evalution}

Our DP algirithm as well as several existing algorithms are implemented in Python, and are compared in terms of the runtime of the algorithms and the quality of solutions (i.e., the energy consumption of the obtained routes). Seven routing algorithms shown below are compared in the experiments.

$T S P-N N$ : The nearest-neighbor algorithm for TSP.

$T S P-B F$ : A brute-force algorithm for TSP.

TSP-DP: A dynamic programming algorithm for TSP [5].

$E M-H F$ : A heviest-first algorithm for EMVRP. It iteratively selects the heaviest item one after another.

$E M-N N$ : An NN-like algorithm for EMVRP. It iteratively selects the minimum-energy neighbor one after another.

$E M-B F$ : A brute-force algorithm for EMVRP.

$E M-D P$ : Our DP algorithm proposed in this paper.

Expression (4), which is presented in Ref. [1], is used for energy calculation. Based on the power measurement results in Ref. [6], $W_{\text {drone }}$ is set to 300 and the maximum payload is set to 48. A total of 320 instances of EMVRP are randomly generated, where the number of customers ranges from 5 to 20 . For each number of customers, there are 20 problem instances.

Figure 2 shows the results on the runtime of the seven routing algorithms on Intel Core i5 processor. The complexity of our TSP-NN, EM-HF and EM-NN implementations are $O\left(N^{2}\right)$, and their runtime is less than 1 millisecond in any cases. The complexity of TSP-BF and EM-BF is $O(N$ !), and they fail to find optimal solutions within an hour for delivery to more than 12 customers. Although the complexity of TSP-DP and EM-DP is exponential, they are practical for delivery to 20 customers.

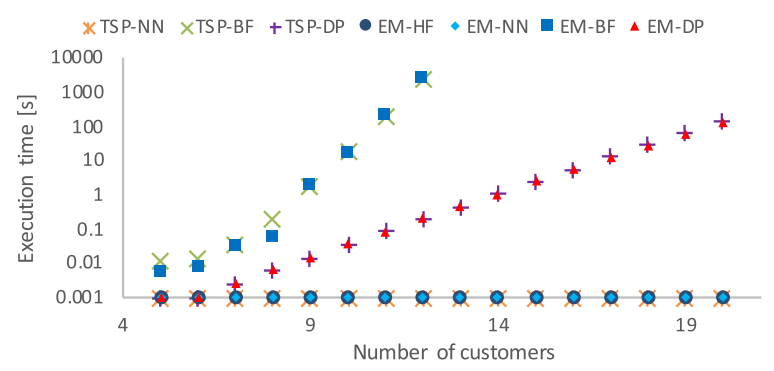

Fig. 2 Runtime of the routing algorithms. 


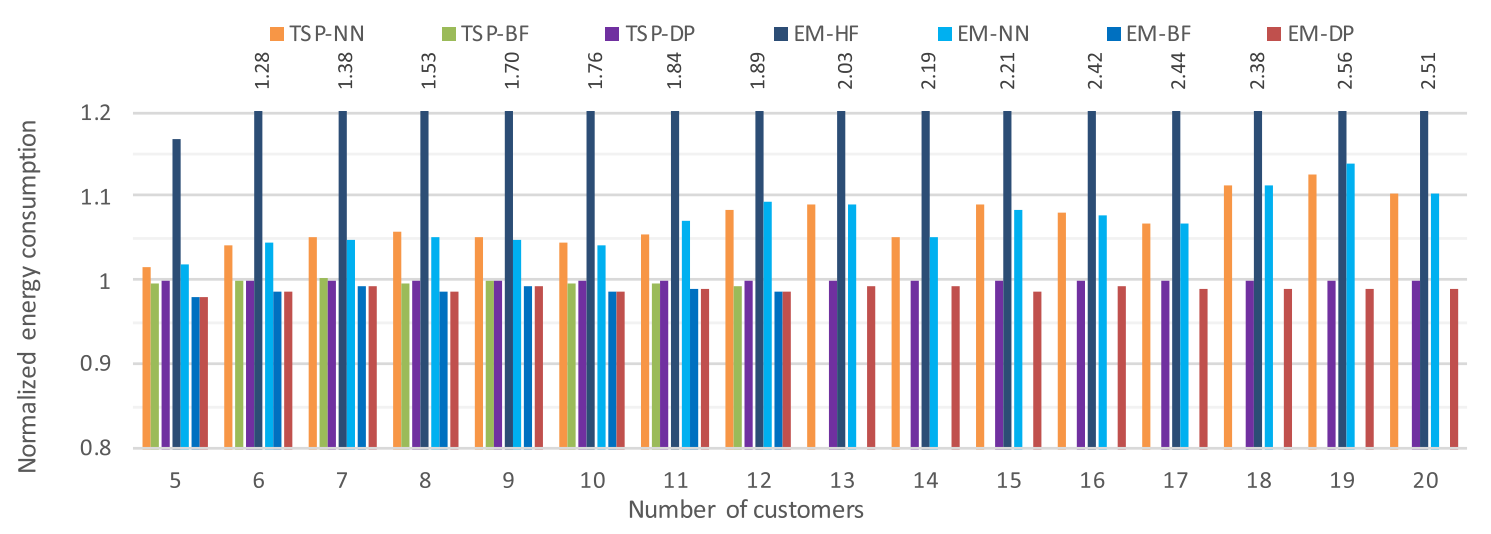

Fig. 3 Energy consumption of the obtained routes.

Figure 3 shows the results on energy consumption obtained by the seven algorithms, where the results are normalized to TSPDP. As mentioned above, there exist 20 problems for each number of customers. In the figure, the average of the seven results is depicted. The results show that the three heuristic algorithms, TSP-NN, EM-HF and EM-NN, are not effective. As long as EM$\mathrm{BF}$ finds solutions, it is confirmed that our EM-DP algorithm successfully finds the same solutions as EM-BF does. Also, it is observed that TSP-DP is not optimal in terms of energy consumption. EM-DP outperforms TSP-DP by $1.08 \%$ on average.

\section{Conclusions}

In this paper, we have proposed a dynamnic programming algorithm for an energy-minimizing routing problem for delivery drones. Experimental results show that the proposed algorithm efficiently finds optimal routes.

The routing problem addressed in this paper is static one, meaning that a set of items to deliver are fixed. In future, we plan to address dynamic problems where delivery orders arrive at the depot next from next over time.

Acknowledgments This work is in part supported by KAKENHI 20H04160.

\section{References}

[1] Kara, I., Kara, B.Y. and Yetis M.K.: Energy Minimizing Vehicle Routing Problem, Proc. International Conference on Combinatorial Optimization and Applications, pp.62-71, Springer (2007).

[2] Negoro, S., Taniguchi, I. and Tomiyama, H.: Fundamental Analysis of Low Energy Path Routing for Delivery Quadcopters, Proc. International Technical Conference on Circuits/Systems, Computers and Communications (2016).

[3] Dorling, K., Heinrichs, J., Messier, G.G. and Magierowski, S.: Vehicle Routing Problems for Drone Delivery, IEEE Trans. Systems, Man, and Cybernetics: Systems, Vol.47, pp.70-85 (2017).

[4] Wang, S. and Liu, X.: Energy Minimization Vehicle Routing Problem with Heterogeneous Vehicles, Proc. International Conference on Service Systems and Service Management (2016).

[5] Held, M. and Karp, R.M.: A Dynamic Programming Approach to Sequencing Problems, Journal of the Society for Industrial and Applied Mathematics, Vol.10, pp.196-210 (1962).

[6] Negoro, S., Sukezane, D., Shibata, A., Maekawa, K., Taniguchi, I. and Tomiyama, H.: Measurement and Modeling of Quadcopter Energy with ROS, Workshop on Synthesis and System Integration of Mixed Information Technologies (2018).

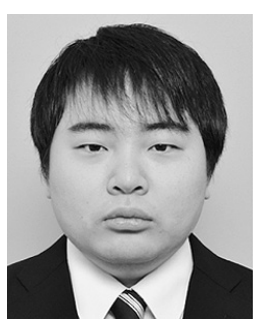

Yusuke Funabashi received his B.E. and M.E. degrees from Ritsumeikan University in 2018 and 2020, respectively. At present, he works for Konami Digital Entertainment. His research interests include mathematical optimization for cyber-physical systems.

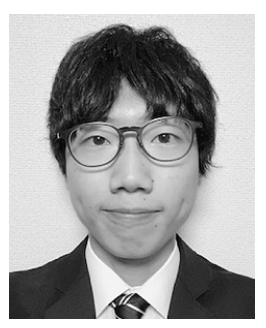

Atsuya Shibata received his B.E. degree from Ritsumeikan University in 2017 and his M.E. degree from Nara Institute of Science and Technology in 2019. At present, he works for Fixstars Corporation. His research interests include algorithm design and software programming.

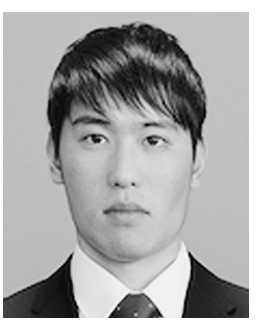

Shunsuke Negoro received his B.E. and M.E. degrees from Ritsumeikan University in 2017 and 2020, respectively. At present, he works for ISHIDA. His research interests include cyber-physical systems.

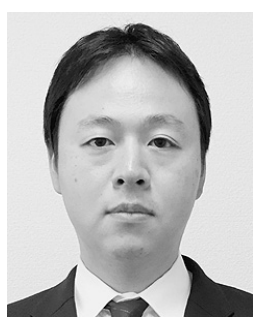

Ittetsu Taniguchi received B.E., M.E., and $\mathrm{Ph} . \mathrm{D}$ degrees from Osaka University in 2004, 2006, and 2009, respectively. From 2007 to 2008, he was an international scholar at Katholieke Universiteit Leuven (IMEC), Belgium. In 2009, he joined the College of Science and Engineering, Ritsumeikan University as an assistant professor, and became a lecturer in 2014. In 2017, he joined the Graduate School of Information Science and Technology, Osaka University as an associate professor. His research interests include system level design methodology, design methodologies for cyber-physical systems, etc. He is a member of IEEE, ACM, IEICE, and IPSJ. 


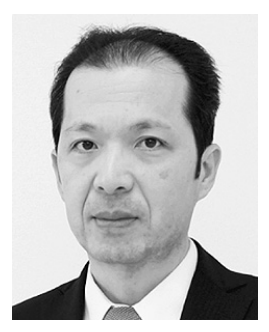

Hiroyuki Tomiyama received his B.E., M.E. and D.E. degrees in computer science from Kyushu University in 1994, 1996 and 1999, respectively. He worked as a visiting researcher at UC Irvine, as a researcher at ISIT/Kyushu, and as an associate professor at Nagoya University. Since 2010, he has been a full professor with College of Science and Engineering, Ritsumeikan University. He has served on program and organizing committees for a number of premier conferences including DAC, ICCAD, DATE, ASP-DAC, CODES+ISSS, CASES, ISLPED, RTCSA, FPL and MPSoC. He has also served as editor-in-chief for IPSJ TSLDM, as an associate editor for ACM TODAES, IEEE ESL and Springer DAEM, and as chair for IEEE CS Kansai Chapter and IEEE CEDA Japan Chapter. His research interests include, but not limited to, design methodologies for embedded and cyberphysical systems.

(Recommended by Associate Editor: Izumi Nitta) 